\title{
Single-shot time-resolved magnetic x-ray absorption at a free-electron laser
}

\author{
Emmanuelle Jal, ${ }^{1, *}$ Mikako Makita, ${ }^{2}$ Benedikt Rösner, ${ }^{2}$ Christian David, ${ }^{2}$ Frithjof Nolting, ${ }^{2}$ Jörg Raabe, ${ }^{2}$ Tatiana Savchenko, ${ }^{2}$ \\ Armin Kleibert, ${ }^{2}$ Flavio Capotondi, ${ }^{3}$ Emanuele Pedersoli, ${ }^{3}$ Lorenzo Raimondi, ${ }^{3}$ Michele Manfredda, ${ }^{3}$ Ivaylo Nikolov, ${ }^{3}$ \\ Xuan Liu, ${ }^{1}$ Alaa el dine Merhe, ${ }^{1}$ Nicolas Jaouen, ${ }^{4}$ Jon Gorchon, ${ }^{5}$ Gregory Malinowski, ${ }^{5}$ Michel Hehn, ${ }^{5}$ \\ Boris Vodungbo, ${ }^{1}$ and Jan Lüning ${ }^{1,4}$ \\ ${ }^{1}$ Sorbonne Université, CNRS, Laboratoire de Chimie Physique - Matière et Rayonnement, LCPMR, 75005 Paris, France \\ ${ }^{2}$ Paul Scherrer Institut, 5232 Villigen PSI, Switzerland \\ ${ }^{3}$ FERMI, Elettra-Sincrotrone Trieste, SS 14 - km 163.5, 34149 Basovizza, Trieste, Italy \\ ${ }^{4}$ Synchrotron SOLEIL, L' Orme des Merisiers, Saint-Aubin, 91192 Gif-sur-Yvette, France \\ ${ }^{5}$ Institut Jean Lamour, Université de Lorraine, Nancy, France
}

(Received 14 November 2018; revised manuscript received 30 January 2019; published 15 April 2019)

\begin{abstract}
Ultrafast dynamics are generally investigated using stroboscopic pump-probe measurements, which characterize the sample properties for a single, specific time delay. These measurements are then repeated for a series of discrete time delays to reconstruct the overall time trace of the process. As a consequence, this approach is limited to the investigation of fully reversible phenomena. We recently introduced an off-axis zone plate based $\mathrm{x}$-ray streaking technique, which overcomes this limitation by sampling the relaxation dynamics with a single femtosecond $\mathrm{x}$-ray pulse streaked over a picosecond long time window. In this article we show that the $\mathrm{x}$-ray absorption cross section can be employed as the contrast mechanism in this novel technique. We show that changes of the absorption cross section on the percent level can be resolved with this method. To this end we measure the ultrafast magnetization dynamics in CoDy alloy films. Investigating different chemical compositions and infrared pump fluences, we demonstrate the routine applicability of this technique. Probing in transmission the average magnetization dynamics of the entire film, our experimental findings indicate that the demagnetization time is independent of the specific infrared laser pump fluence. These results pave the way for the investigation of irreversible phenomena in a wide variety of scientific areas.
\end{abstract}

DOI: 10.1103/PhysRevB.99.144305

\section{INTRODUCTION}

Femtosecond time-resolved experiments employing advanced $\mathrm{x}$-ray probe techniques have been realized in a wide variety of scientific domains since the advent of ultrashort $\mathrm{x}$-ray-pulse sources such as femtoslicing, high harmonic generation, and x-ray free-electron lasers (XFELs). Research activities range from the investigation of fundamental processes in gas phase experiments [1-3] to the observation of structural rearrangement in biological macromolecules using novel time-resolved crystallography techniques $[4,5]$. In condensed matter physics, the availability of ultrahort $\mathrm{x}$-ray pulses has allowed us to probe ultrafast charge, spin, and lattice dynamics with chemical selectivity and nanometer spatial resolution [6-10].

A significant number of these time-resolved experiments investigate the relaxation dynamics following an externally induced excitation, which is typically realized by an ultrashort infrared (IR) pump pulse. To characterize these dynamics, the probe signal is generally obtained by accumulating statistics over repetitive pump-probe cycles for a specific delay. The advent of XFELs promised to overcome this need of signal accumulation due to their high pulse intensity, their temporal coherence, their femtosecond pulse duration, and due to the strong sensitivity of $\mathrm{x}$-ray probe techniques. The feasibility

*emmanuelle.jal@sorbonne-universite.fr of such x-ray single-shot probing has indeed been demonstrated [11]. Nevertheless, the pump-probe cycle has to be repeated for different time delays to reconstruct the overall time evolution of the relaxation dynamics from these individual measurements. As a consequence, identical experimental conditions must be re-established for each pump-probe cycle, which may be compromised due to practical aspects such as the reproducibility of the pump and probe parameters. In addition, temporal jitter between pump and probe pulses limits the achievable time resolution in such experiments. Finally, a fundamental restriction concerns the reversibility of the excitation process itself and of the sample's initial state, limiting the application of pump-probe techniques to the investigation of fully reversible ultrafast dynamics.

We recently demonstrated a novel experimental approach, which overcomes these restrictions by continuous probing of a relaxation process with a single x-ray pulse [12]. For this we employ an off-axis Fresnel zone plate to stretch an incoming $x$-ray pulse while introducing at the same time an angular encoding of the arrival time of the $\mathrm{x}$ rays. In our previous work we have performed such an x-ray streaking experiment in a reflection geometry and used the resonant transverse magneto-optic Kerr effect (T-MOKE) to follow the ultrafast magnetization dynamics in a thin transition metal film [12]. Since resonant T-MOKE provides a very strong magnetic dichroism (40\% in our case [12]), it was ideally suited for a first feasibility demonstration. On the other hand, this experimental geometry can only be employed to study 
quantitatively the magnetization dynamics, and is limited to probing surface phenomena in materials exhibiting an inplane magnetization.

To demonstrate a broader applicability of this X-ray streaking technique we are reporting here a further development where an off-axis zone plate is used to generate a tilted wave front as a probe for $\mathrm{x}$-ray absorption spectroscopy. Specifically, we use the $\mathrm{x}$-ray magnetic circular dichroism (XMCD) effect $[13,14]$ in transmission to follow the magnetization dynamics of Co ( $M$ edges) in thin CoDy alloy films exhibiting out-of-plane magnetization. Our results demonstrate that signal variations of a few percent level can be resolved. It suggests that an absorption spectroscopy based x-ray streaking is suitable for the investigation of ultrafast dynamics in a variety of other fields [15-17].

Since the discovery of laser-induced ultrafast demagnetization by Beaurepaire et al. [18], intense research to understand what is driving this phenomenon have been performed, leading to another discovery of technological relevance: the possibility of single-shot all optical switching in transition metal (TM)-rare earth (RE) alloys [19]. In order to better understand what is the influence of the RE elements on the dynamics of the TM, we have probed the Co magnetization dynamics for two CoDy alloys with different chemical compositions. In this study we find that for higher Dy concentration the initial quenching observed at the Co site is slower, but reaches a higher degree of demagnetization. Furthermore, for each alloy, varying the pump fluence does not affect the timescale of the initial magnetization quenching.

\section{EXPERIMENT}

The experiment has been realized at the DiProI end station [20] of the seeded XUV-FEL FERMI at Elettra, Trieste [21], which provides femtosecond short X-ray pulses with full polarization control. The experimental layout of our zone plate based X-ray streaking experiment is shown in Fig. 1(a). The off-axis zone plate is the same as used in our previous experiment [12]. To overfill its $4.84 \mathrm{~mm}$ square aperture, the beamline focus was moved far behind the sample position by releasing the bend of the $\mathrm{KB}$ mirrors, producing a nearly collimated beam. The converging positive first order of the zone plate passes through the sample and its intensity distribution is collected by a CCD camera (so-called sample camera afterwards). Note that the photons detected at the bottom end of the sample camera [yellow line in Fig. 1(a)] have traveled a longer path than those arriving at the top of the camera [green line in Fig. 1(a)]. Their arrival time difference at the zone plate focus $(\Delta t=1.57 \mathrm{ps})$ is proportional to the product of the number of zone pairs $(23000)$ and the $x$-ray wavelength $(20.5 \mathrm{~nm})$. The time evolution of the measured signal is therefore given by the intensity variations between those two points.

The sample is positioned in the vicinity of the focus of the positive first diffraction order. This allows for controlling the X-ray fluence on the sample to avoid x-ray induced modifications, while limiting the different optical path, i.e., time delays, to probe different sample areas. A precise calibration of the time axis is obtained by varying the delay between IR and $\mathrm{x}$-ray pulses. A permanent magnetic field of $\pm 250 \mathrm{mT}$ is

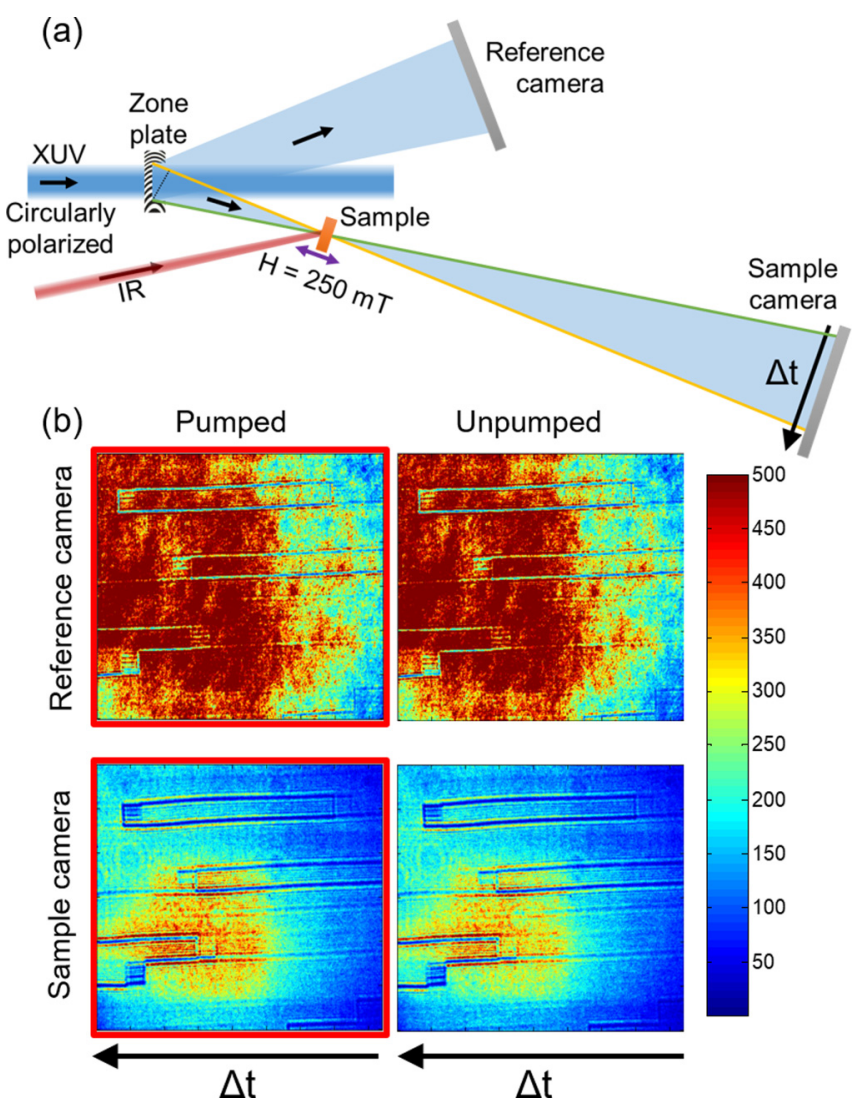

FIG. 1. (a) Sketch of the experimental setup. (b) Intensity distribution of a single $\mathrm{x}$-ray pulse recorded by the sample camera (converging first order, after passing through the $\mathrm{Co}_{76} \mathrm{Dy}_{24}$ thin film sample) and reference camera (diverging first order) with (pumped) and without (unpumped) IR laser excitation.

applied to reset a single domain state after each pump-probe event.

To characterize the shot to shot variations of the spatial intensity distribution of incident x-ray pulse, a second CCD camera (reference camera) is used to record the diverging negative first order. For a single x-ray pulse, two images are therefore recorded simultaneously: the signal characterizing the observed dynamics is encoded in the intensity variation of the sample camera image; and the normalization with respect to the incident intensity distribution is provided by the simultaneously recorded image of the reference camera. The higher diffraction orders of the diffractive element are spatially filtered along the beam path from the zone plate to the detector in order to minimize their contamination at the sensor plane. The sample to signal CCD distance has been chosen such that the pixel size does not limit the achievable time resolution. The zone plate to reference CCD distance was then chosen such that comparable magnifications are obtained for both cameras, which simplifies data analysis. Final adjustment of the image sizes is done by a spatial image transformation. Aluminum filters have been placed in front of both cameras to block the IR transmitted/scattered after the sample. In order to get the same XUV contrast on both cameras, the $\mathrm{Al}$ filter in front of the reference camera is much thicker than the one in front of the sample camera. 
(a) 500 shots

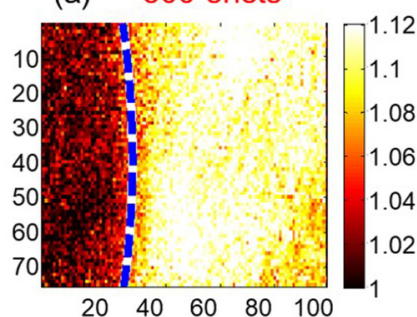

(b) Single shot

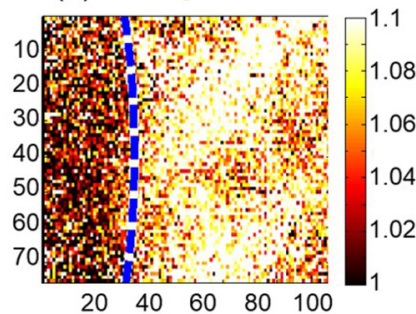

(c)

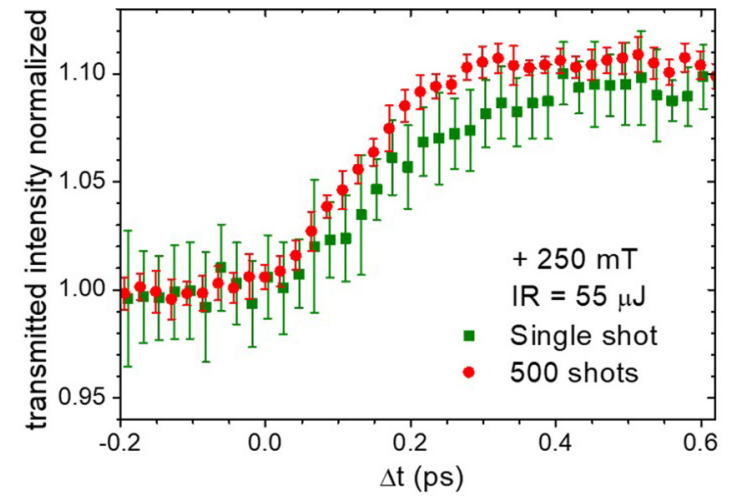

FIG. 2. Treated transmission signal image (see text) revealing the IR-pulse-induced magnetization dynamics of a thin $\mathrm{Co}_{76} \mathrm{Dy}_{24}$ film in the presence of an out-of-plane applied field of $250 \mathrm{mT}$ and an IR pump pulse intensity of $55 \mu \mathrm{J}$. (a) The signal of 500 pump-probe cycles was accumulated to obtain a high signal-to-noise image. The dashed blue line indicates pump-probe time overlap. (b) Signal image obtained from a single pump-probe event. (c) Azimuthally integrated radial intensity of the signal images projected onto the time delay axis (red for 500 shots, green for single shot) normalized by the unpumped intensity.

The images in Fig. 1(b) show two pairs of single x-ray pulse images recorded by the reference (top line) and sample camera (bottom line). The two images in the left column were recorded with IR pulse excitation (pumped), while no excitation was applied in the case of the right column (unpumped). Comparing the pumped and unpumped raw images, there are no clear evidences of a pump pulse induced evolution of the magnetization. However, as demonstrated in the next section, starting from these four images an appropriate normalization procedure yields an image that clearly reveals the signature of the laser-pulse-induced ultrafast magnetization dynamics (Fig. 2).

The measurements reported in this article have been performed on 50-nm-thick $\mathrm{Co}_{1-x} \mathrm{Dy}_{x}$ alloy films, which were deposited by magnetron sputtering on $30-\mathrm{nm}$-thin $\mathrm{Si}_{3} \mathrm{~N}_{4}$ membranes. A seed layer of $3 \mathrm{~nm}$ of Ta and $5 \mathrm{~nm}$ of Pt was used and the film was capped with a 5-nm-thick Pt layer to prevent oxidation. Two different alloy compositions were investigated: $\mathrm{Co}_{86} \mathrm{Dy}_{14}$ and $\mathrm{Co}_{76} \mathrm{Dy}_{24}$. At room temperature, both films exhibit a magnetic out-of-plane anisotropy and a square hysteresis loop with a coercive field of $100 \mathrm{mT}$ for $\mathrm{Co}_{86} \mathrm{Dy}_{14}$ and $200 \mathrm{mT}$ for $\mathrm{Co}_{76} \mathrm{Dy}_{24}$.

To realize jitter free IR-pump/XUV-probe measurements, a fraction of FERMI's IR seed laser was used to excite the sample. As shown previously [20], this yields sub-10-fs jitter between IR and XUV pulse arrival time and repetitive, ac- cumulative measurements can be realized without significant degradation of the overall time resolution. The wavelength of the linearly polarized IR pulses was centered at $780 \mathrm{~nm}$ and the pulse length is about $100 \mathrm{fs}$. The IR laser spot size at the sample position was estimated, thanks to phosphor paint, to be about $500 \times 400 \mu \mathrm{m}^{2}$ (FWHM). To reach the typical optical intensity range inducing sizable effects on magnetic response an IR pulse from 5.5 to $55 \mu \mathrm{J}$ was used.

The photon energy of the circularly polarized XUV pulses was tuned to $20.5 \mathrm{~nm}$ to match the magnetically dichroic $M_{2,3}$ absorption edge of Co (see note [22]). Using the 12th harmonic of the seed laser, the XUV pulse length is expected to be about $70 \mathrm{fs}$ [23]. The XUV spot size in the sample plane was measured to be $\approx 150 \times 100 \mu \mathrm{m}^{2}$ (FWHM), thus probing a homogeneous part of the IR pumped area. The XUV intensity at the sample plane was set far below the excitation regime [11].

\section{RESULTS}

The as-recorded images shown in Fig. 1 exhibit very strong intensity fluctuations, which outweigh the signal variations from the IR-excitation-induced magnetization dynamics. These fluctuations have three origins: the spatially inhomogeneous intensity distribution of the incident XUV pulse, inhomogeneities in the XUV diffraction of the zone plate, and the thickness variations of the $\mathrm{Al}$ filter protecting the $\mathrm{CCD}$ cameras against the IR photons of the pump pulse. These filter thickness variations, in particular for the thicker Al filter of the reference camera, introduce substantial differences between the pattern recorded by sample and reference cameras. In addition, the zone plate diffracted images show artifacts, such as horizontal streaks due to stitching errors from the zone plate fabrication process. These stitching errors give rise to phase contrast in the far field plane of both CCD cameras. The image recorded by the reference camera can therefore be used to correct these artifacts in the image recorded by the sample camera.

To extract the signature of the magnetization dynamics from the pumped image, we have to employ background images recorded by the two cameras without XUV probe pulse, and with and without IR pump pulse. These images have to be recorded each time there is any change in experimental parameters. A complete data set is thus composed of eight images, the four shown in Fig. 1 and four background pictures without XUV probe pulse (not shown). The background count levels in these images are different for both cameras as well as when the IR pump is used or not. We therefore need both backgrounds, with and without IR. The applied procedure to extract the desired signal consists of first background correcting each XUV camera image and then normalizing for each camera the pumped by the corresponding unpumped image. This normalized image of the sample camera is then divided by the one of the reference camera to get the final signal map, whose intensity variation exhibits the IR-pulse-induced magnetization dynamics.

Final signal maps obtained by this procedure are shown in Fig. 2. These are recorded by transmission of the XUV probe pulse through a thin $\mathrm{Co}_{76} \mathrm{Dy}_{24}$ film under the presence of an out-of-plane magnetic field of $250 \mathrm{mT}$. The IR pump 
pulse intensity was $55 \mu \mathrm{J}$. The image in Fig. 2(a) is obtained by accumulating the signal for 500 pump-probe cycles, while a single pump-probe cycle yielded the image shown in Fig. 2(b). The pump-probe delay axis proceeds mainly in the horizontal direction from left to right. The IR-pulse-induced magnetization dynamics is clearly visible in both images as an abrupt color change, which sets in right after temporal overlap between IR pump and XUV probe pulses (dashed blue line).

All $\mathrm{x}$ rays coming from the same zone pair of the off-axis zone plate reach the sample at the same time and therefore probe the same delay. On the CCD camera, which records a magnified image of the off-axis zone plate, these intensities lay on the curved trace of the corresponding zone pair and can thus be averaged by an angular integration (e.g., along the dashed blue line for zero delay). This procedure yields the projection of the signal onto the pump-probe time delay axis, as shown in Fig. 2(c) for the accumulation of 500 shots (red) and the single-shot measurement (green). These intensities are normalized by the unpumped signal and are therefore equal to 1 before time 0 . The error bars are determined from the standard deviation of the binning of the temporal axis. The good signal to noise ratio of the single-shot measurement underlines the potential of this technique. In this particular configuration of circular polarization and magnetic field orientation, the laser-induced demagnetization leads to a signal increase reflecting the expected rapid quenching of the magnetization.

The two delay curves exhibit overall identical shapes, which underlines the reversibility of the laser-induced demagnetization process. The slightly lower degree of demagnetization observed in the single-shot measurement suggests a lower IR pump fluence. This may be caused by shot-to-shot fluctuations of the pump laser intensity, or by pointing fluctuations of the XUV probe pulse altering the probed sample area with respect to the IR pump pulse. Note that single-shot measurements overcome these limitations, emphasizing the relevance of this technique even when studying reversible processes.

Measuring magnetization dynamics for opposite direction of externally applied magnetic field allows us to quantify the degree of the demagnetization dynamic. Figure 3(a) shows the result of such a measurement, non-normalized by the unpumped signal, which has been performed on a $\mathrm{Co}_{76} \mathrm{Dy}_{24}$ film by averaging 500 pump-probe cycles and an IR pump pulse intensity of $55 \mu \mathrm{J}$. The blue and red curves show the temporal evolution of the transmitted XUV intensity for opposite outof-plane field directions. The logarithmic of these curves give the absorption $\mu^{ \pm}$, and the difference between the two absorptions $\mu^{-}$and $\mu^{+}$gives the XMCD intensity [Fig. 3(b)] [24], which is directly proportional to the film's magnetization [25]. For negative delays, the separation between those two curves represents the unperturbed magnetization of the film's ground state, while the laser-induced ultrafast demagnetization leads to a reduction of this separation. In order to disentangle the electronic and magnetic dynamic of the film we use conventional analysis for dichroic response: where the average curve [shown by the black line in Fig. 3(a)] takes into account the electronic excitation while the logarithm of the ratio of those two curves collected with different orientation of the magnetic field, i.e., XMCD signal, represent the magnetiza-

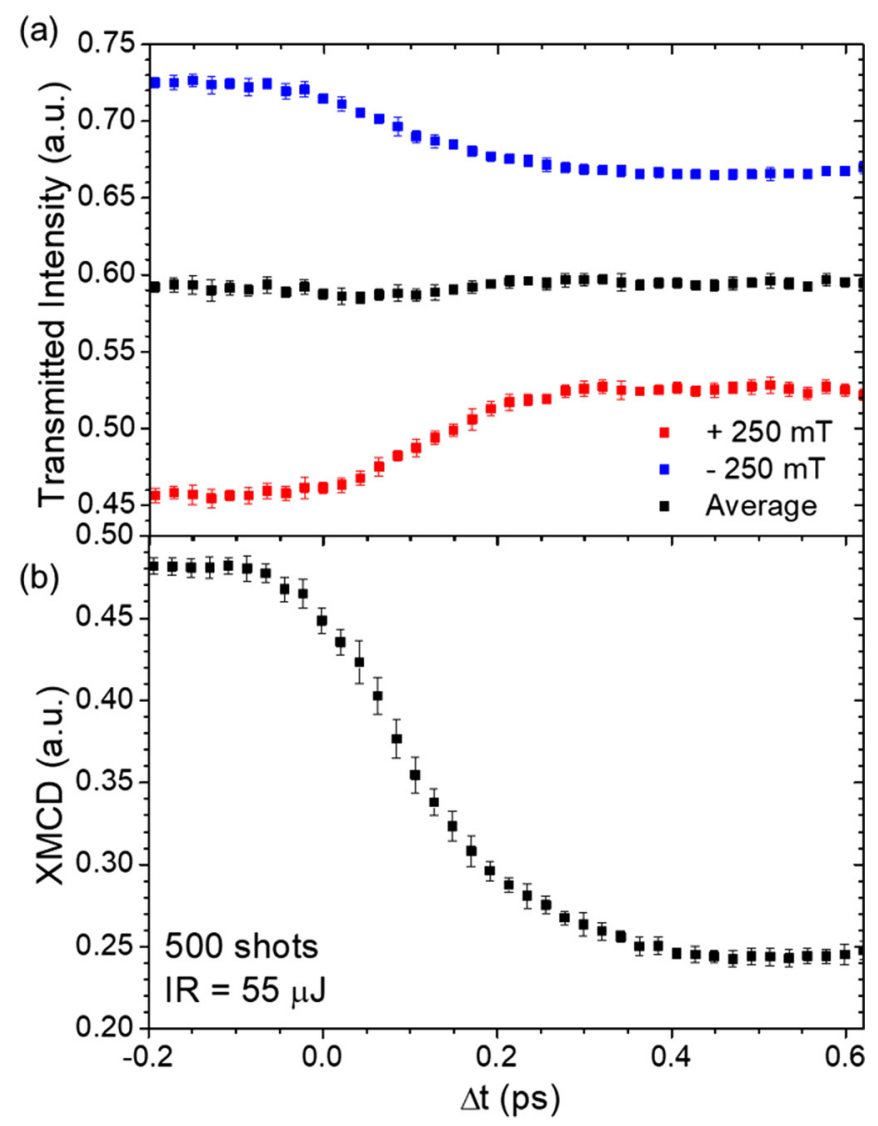

FIG. 3. (a) Non-normalized transmitted intensity as a function of pump-probe time delay recorded on a $\mathrm{Co}_{76} \mathrm{Dy}_{24}$ film by averaging 500 pump-probe cycles (IR intensity of $55 \mu \mathrm{J}$ ). A static out-of-plane magnetic field of $250 \mathrm{mT}$ was applied in positive (red) and negative (blue) direction. The black curve shows the arithmetic average of the two signals. (b) The logarithm of the ratio of the blue and red curves in (a) gives the temporal evolution of the XMCD signal.

tion dynamic [Fig. 3(b)]. The nearly constant value of the average curve in Fig. 3(a) shows that in this pumping regime the electronic response is almost negligible, while most of the sample response is due to the reduction of the XMCD signal. The laser-pulse-induced reduction of the XMCD amplitude indicates a demagnetization degree of about $50 \%$.

Looking at the noise level of the single-shot data in Fig. 2(c) we can quantify our experimental detection limit to correspond to a $2 \%$ change in the transmitted intensity, which highlights the high sensitivity of this single-shot technique. For multiple data exposure, the detection limit can be as low as $0.7 \%$ [26].

The normalized demagnetization curves recorded on $\mathrm{Co}_{76} \mathrm{Dy}_{24}$ and $\mathrm{Co}_{84} \mathrm{Dy}_{16}$ for different IR pump fluence values are shown in Fig. 4. To facilitate the comparison we plot the degree of demagnetization $\left(\Delta M / M_{0}\right)$, which increases as expected with increasing pump fluence for each film. Comparing the results obtained for the two films we note that the film with higher Co concentration $\left(\mathrm{Co}_{84} \mathrm{Dy}_{16}\right)$ exhibits a weaker demagnetization for equivalent IR pump intensity. Note that the relative IR pump-XUV probe alignment was verified in both cases. The relationship between IR pump fluence and achieved degree of demagnetization is shown in Fig. 4(c). The 

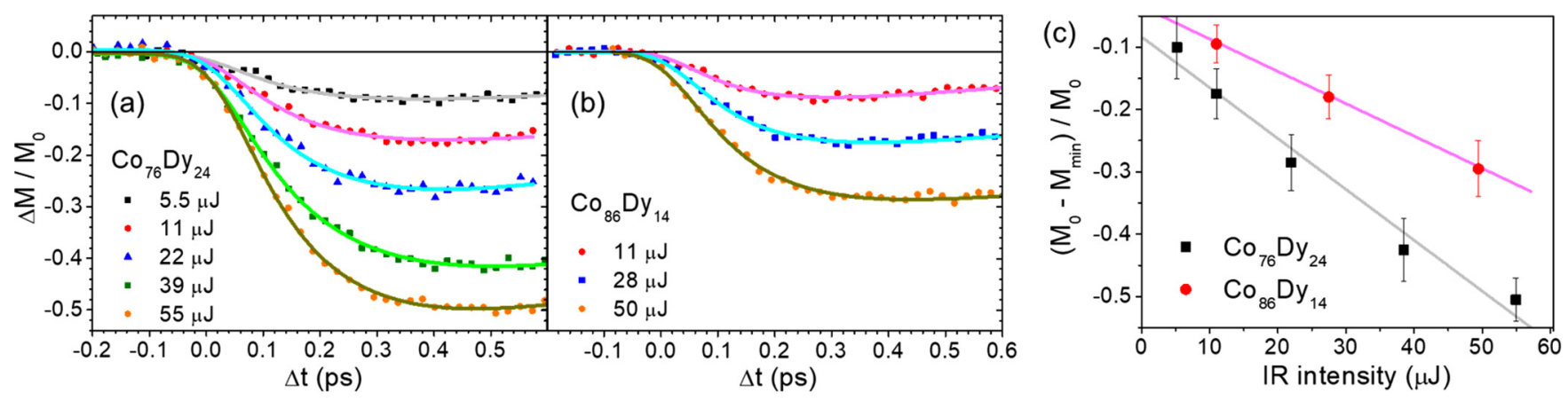

FIG. 4. Normalized demagnetization dynamics as a function of pump fluence recorded (a) on $\mathrm{Co}_{76} \mathrm{Dy}_{24}$ (500 shots accumulated) and (b) on $\mathrm{Co}_{86} \mathrm{Dy}_{14}$ (50 shots accumulated). (c) Degree of demagnetization as a function of the employed IR laser fluence for $\mathrm{Co}_{76} \mathrm{Dy}_{24}$ (black squares) and $\mathrm{Co}_{86} \mathrm{Dy}_{14}$ (red circles). The lines are a linear fit.

achieved degree of demagnetization increases for each film linearly with IR pump fluence over the sampled range of up to $50 \%$ of demagnetization, which is in agreement with previous studies on other Co containing TM-RE alloys [27-29]. The above mentioned lower degree of demagnetization for higher Co concentration is reflected by the different slopes.

To extract parameters from those demagnetization curves, we have fitted all data with a two exponential model [30]:

$$
\frac{d m}{d t}=\Gamma(t) \otimes\left\{a+\Theta(t)\left[b+c * e^{-\frac{t}{\tau_{m}}}-d * e^{-\frac{t}{\tau_{e}}}\right]\right\} .
$$

In this equation $\Gamma(t)$ is the experimental time resolution given by a Gaussian function taking for FWHM the laser and FEL pulse duration added in quadrature, $\Theta(t)$ is the step function, $a$ represents the magnetization value before time zero $\left(t_{0}\right)$, $\tau_{m}$ represents the characteristic demagnetization time, and $\tau_{e}$ is the characteristic remagnetization time. Since the sampled time window extends only to $0.6 \mathrm{ps}$ after $t_{0}$ [31], we have, for each measurement, set the remagnetization parameter $\tau_{e}$ to a value taken from time-resolved MOKE measurements (not shown).

The experimental data are very well reproduced by these fits, which are shown by the lines in Fig. 4. Within the uncertainty of this analysis, we find that for each film the demagnetization time does not depend on the employed IR pump fluence. The obtained values are $140 \pm 20$ fs for $\mathrm{Co}_{76} \mathrm{Dy}_{24}$ and $115 \pm 20$ fs for $\mathrm{Co}_{86} \mathrm{Dy}_{14}$, which is in good agreement with the result of Ferté et al. [32] obtained on similar alloy composition $\left(\mathrm{Co}_{78} \mathrm{Dy}_{22}\right)$.

This numerical result can be confirmed visually by scaling the demagnetization curves, measured for different pump fluences, to equal amplitude as shown for $\mathrm{Co}_{76} \mathrm{Dy}_{24}$ in Fig. 5(a). The absence of any systematic deviation between these data underlines that the IR pump fluence does not alter the observed demagnetization time. Figure 5(b) shows the average of those scaled curves for all the IR fluences, for $\mathrm{Co}_{76} \mathrm{Dy}_{24}$ (black) and $\mathrm{Co}_{86} \mathrm{Dy}_{14}$ (red), emphasizing the presence of a different demagnetization timescale for different chemical composition.

\section{DISCUSSION}

Since the discovery of ultrafast magnetization dynamics [18], intense research efforts have been made to reveal the underlying mechanism driving the ultrafast demagnetization [6,7,30,33-44]. While these studies report overall very similar magnetization dynamics, one notices differences in details such as the specific characteristic timescale of magnetization quenching and partial recovery. These discrepancies may be sample structure related, but there are also indications that differences in probing depth of the employed techniques may play a role $[45,46]$. It is in this regard important to note that in our transmission experiment the entire magnetic layer is probed. This implies that we are probing the average

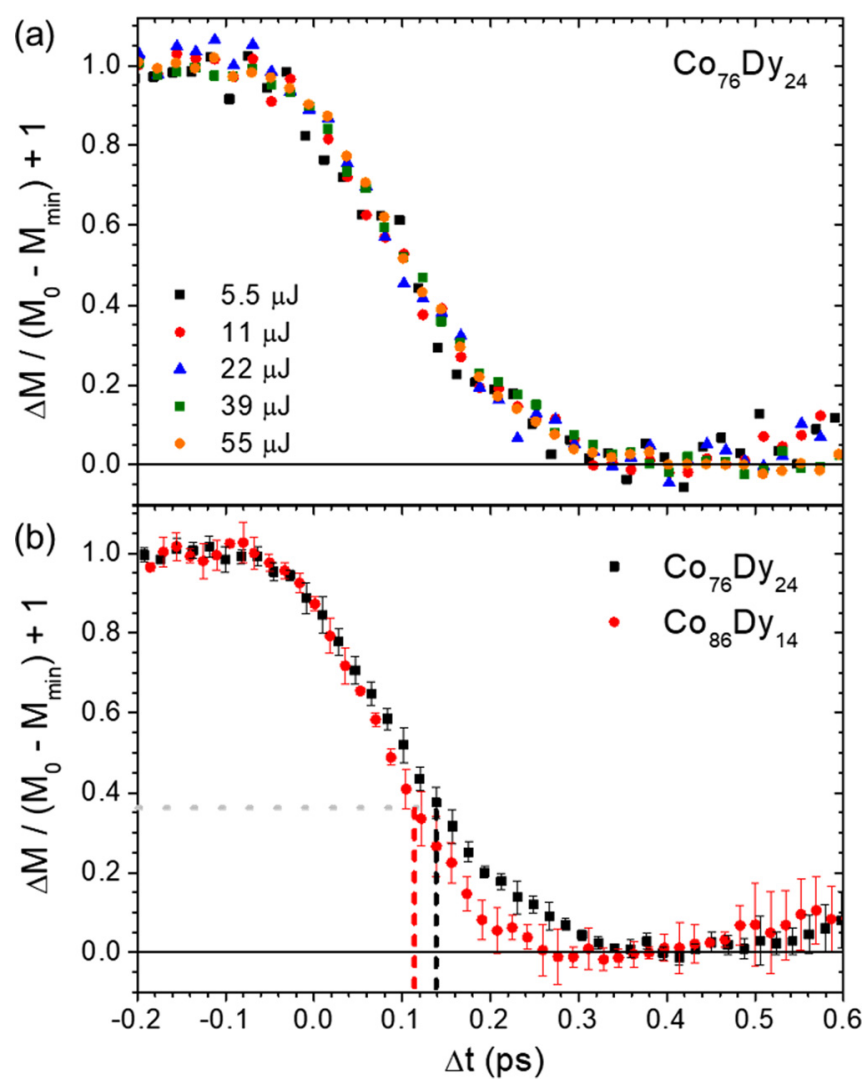

FIG. 5. (a) Scaled demagnetization curves recorded on $\mathrm{Co}_{76} \mathrm{Dy}_{24}$ for different IR fluence. (b) A comparison between the scaled curves of $\mathrm{Co}_{76} \mathrm{Dy}_{24}$ and $\mathrm{Co}_{86} \mathrm{Dy}_{14}$ (average of the respective fluence data) highlights the slower demagnetization time of the Dy rich alloy $\left(\mathrm{Co}_{76} \mathrm{Dy}_{24}\right)$. 
magnetization dynamics, regardless of the excitation profile. For our films with a thickness of $50 \mathrm{~nm}$ (chosen to provide a strong XMCD signal) this implies that we are exciting mostly the first quarter of the magnetic film, whereas we are probing the entire sample depth. This has to be kept in mind when comparing our results to other studies which are either looking at thinner films [32], or with more surface sensitive probing techniques.

First, our data indicate that degree and timescale of the magnetization quenching depends on the chemical composition in CoDy alloys, thereby confirming previous results on TM-RE alloys. For example, Alebrand et al. [27] show that the efficiency of the magnetization quenching by optical excitation in the TM-TE ferrimagnets $\mathrm{Tb}_{x} \mathrm{Co}_{1-x}$ decreases with increasing TM content. This variation of the magnetization quenching with sample composition is related to the Curie temperature dependence on the alloy composition. From the study of Hansen et al. [47] we know that the Curie temperature of $\mathrm{Co}_{76} \mathrm{Dy}_{24}$ is lower than the one of the $\mathrm{Co}_{86} \mathrm{Dy}_{14}$. Exciting both samples at room temperature, it means that for the same amount of deposited energy, the $\mathrm{Co}_{76} \mathrm{Dy}_{24}$ alloy reaches a temperature closer to the Curie temperature. Moreover, the dependence of the degree of magnetization quenching on the fluence can be related to the fact that for longer time delays, the remaining magnetization is proportional to the newly established equilibrium temperature [48]. Increasing the pump fluence increases this new equilibrium temperature and so the transient magnetization is smaller. The observation of a linear dependence indicates that over the employed fluence range the system remains far from the Curie temperature $[49,50]$.

The lower Curie temperature of the Dy rich sample can also explain the observed slower demagnetization time of the $\mathrm{TM}$ in the CoDy ferrimagnet alloy. Indeed as pointed out by Suarez et al. [51] and Atxitia et al. [52] the complex demagnetization dynamic of ferrimagnetic films is strongly related to the concentration of the RE element that mediate the intersublattice exchange strength. In a phenomenological description, the numerical and analytical solutions of the Landau-Lifthitz-Bloch equation $[51,52]$ show that in such systems the demagnetization time of the TM element increases as a function of the RE concentration, assuming that the temperature remains sufficiently far from the Curie temperature. This consideration is in agreement with what we observe experimentally. In Fig. 5(b) one notices that when probing the magnetization dynamics of the TM element a slower quenching is observed for the Dy rich alloy $\left(\mathrm{Co}_{76} \mathrm{Dy}_{24}\right)$. We remark that the different compensation temperatures of our two CoDy alloys seem not to play a role here as demonstrated previously by Ferté et al. [32].

Second, our results clearly demonstrate that for a given CoDy alloy the demagnetization time does not depend on the excitation fluence. This is in agreement with previous studies in which we employed resonant magnetic small angle x-ray scattering $[8,38]$ as a probe technique. However, this finding is different from results of all-optical pump-probe experiments, which reveal a clear dependency of the demagnetization time on the pump fluence [35,53-55]. As mentioned above, a likely explanation could be the different sampling depth of the employed techniques, which is particularly significant in the case of our thick film samples. While in all-optical experiments, the dynamics are usually probed in reflection, implying that the dynamics are sampled with exponentially decreasing sensitivity into the bulk of the film; the entire film is probed in our transmission geometry. Our findings therefore support recent considerations that the probing depth of the employed techniques should be taken into consideration [46].

The observed fluence independency of the demagnetization time can be understood within the model put forward by Suarez et al. [51] and Atxitia et al. [52]. Their model predicts only a week fluence dependence of the demagnetization time as long as the system is not heated above the Curie temperature, which is true in our case, as discussed above. On the other hand, when the temperature reaches or overcomes the Curie temperature, the situation changes as reported by simulation [51,52] and recent experimental work [32,46].

\section{CONCLUSIONS}

In conclusion, $\mathrm{x}$-ray streaking in transmission geometry has been demonstrated by performing single-shot based timeresolved XMCD spectroscopy. This new experimental technique is sensitive to signal change as small as $2 \%$ and opens the path for the investigation of the dynamics of irreversible phenomena in a wide variety of fields.

Our study on CoDy alloys reveals that, for 50-nm-thick films, when the average magnetization dynamics over the entire film thickness is probed, the demagnetization time does not depend on the amount of energy deposited in the sample, but seems to be intrinsically given by the alloy composition.

Further development of the employed zone plate optic holds significant potential to further increase the capabilities of this novel technique. First of all, improving the manufacturing process will reduce the artifacts currently hampering the achievable signal quality. Indeed, we are now able to optimize the fabrication conditions in a way to suppress stitching artifacts almost completely. In addition, the fabrication of off-axis zone plates with finer structures will enable to extend the time window. For example, the current capabilities to manufacture, in a reasonable amount of time, efficient off-axis zone plate optics for time-streaking experiments in the EUV will allow us to access time windows up to $3.3 \mathrm{ps}$ at the iron $M$ edge $(55 \mathrm{eV})$. Last but not least, more complex zone plate schemes are currently designed to extend the scientific possibilities. Specifically, we plan to fabricate off-axis zone plates with (i) two focus spots to have a beam copy transmitted through an unpumped region of the sample and projected onto the same camera for a reliable shot-to-shot reference with the same magnification, and (ii) a segmentation of our zone plate patterns to probe several absorption edges simultaneously.

\section{ACKNOWLEDGMENTS}

The authors are grateful for financial support received from the following agencies: The French Agence National de la Recherche via Projects No. UMAMI ANR-15-CE240009, the CNRS-PICS program, the CNRS-MOMENTUM program, and the SNSF project (No. 200021_160186). Access to Synchrotron SOLEIL through proposal ID 20160880 for characterization of static properties of the CoDy films is 
acknowledged. Furthermore, this project has received funding from the EU-H2020 research and innovation programme under Grant Agreement No. 654360 NFFA-Europe. Finally,
M.M. acknowledges the support from the European Community's Seventh Framework Programme (FP7/20072013) under Grant Agreement No. 290605 (PSI-FELLOW/COFUND).
[1] R. J. Squibb, M. Sapunar, A. Ponzi, R. Richter, A. Kivimäki, O. Plekan, P. Finetti, N. Sisourat, V. Zhaunerchyk, T. Marchenko, L. Journel, R. Guillemin, R. Cucini, M. Coreno, C. Grazioli, M. Di Fraia, C. Callegari, K. C. Prince, P. Decleva, M. Simon, J. H. D. Eland, N. Došlić, R. Feifel, and M. N. Piancastelli, Nat. Commun. 9, 63 (2018).

[2] A. Rudenko, L. Inhester, K. Hanasaki, X. Li, S. J. Robatjazi, B. Erk, R. Boll, K. Toyota, Y. Hao, O. Vendrell, C. Bomme, E. Savelyev, B. Rudek, L. Foucar, S. H. Southworth, C. S. Lehmann, B. Kraessig, T. Marchenko, M. Simon, K. Ueda, K. R. Ferguson, M. Bucher, T. Gorkhover, S. Carron, R. Alonso-Mori, J. E. Koglin, J. Correa, G. J. Williams, S. Boutet, L. Young, C. Bostedt, S.-K. Son, R. Santra, and D. Rolles, Nature (London) 546, 129 (2017).

[3] L. Young, E. P. Kanter, B. Krässig, Y. Li, A. M. March, S. T. Pratt, R. Santra, S. H. Southworth, N. Rohringer, L. F. DiMauro, G. Doumy, C. A. Roedig, N. Berrah, L. Fang, M. Hoener, P. H. Bucksbaum, J. P. Cryan, S. Ghimire, J. M. Glownia, D. A. Reis, J. D. Bozek, C. Bostedt, and M. Messerschmidt, Nature (London) 466, 56 (2010).

[4] N. Coquelle, M. Sliwa, J. Woodhouse, G. Schiró, V. Adam, A. Aquila, T. R. M. Barends, S. Boutet, M. Byrdin, S. Carbajo, E. De la Mora, R. B. Doak, M. Feliks, F. Fieschi, L. Foucar, V. Guillon, M. Hilpert, M. S. Hunter, S. Jakobs, J. E. Koglin, G. Kovacsova, T. J. Lane, B. Lévy, M. Liang, K. Nass, J. Ridard, J. S. Robinson, C. M. Roome, C. Ruckebusch, M. Seaberg, M. Thepaut, M. Cammarata, I. Demachy, M. Field, R. L. Shoeman, D. Bourgeois, J.-P. Colletier, I. Schlichting, and M. Weik, Nat. Chem. 10, 31 (2017).

[5] E. Tayeb-Fligelman, O. Tabachnikov, A. Moshe, O. Goldshmidt-Tran, M. R. Sawaya, N. Coquelle, J.-P. Colletier, and M. Landau, Science 355, 831 (2017).

[6] C. E. Graves, A. H. Reid, T. Wang, B. Wu, S. de Jong, K. Vahaplar, I. Radu, D. P. Bernstein, M. Messerschmidt, L. Müller, R. Coffee, M. Bionta, S. W. Epp, R. Hartmann, N. Kimmel, G. Hauser, A. Hartmann, P. Holl, H. Gorke, J. H. Mentink, A. Tsukamoto, A. Fognini, J. J. Turner, W. F. Schlotter, D. Rolles, H. Soltau, L. Strüder, Y. Acremann, A. V. Kimel, A. Kirilyuk, T. Rasing, J. Stöhr, A. O. Scherz, and H. A. Dürr, Nat. Mater. 12, 293 (2013).

[7] I. Radu, K. Vahaplar, C. Stamm, T. Kachel, N. Pontius, H. A. Dürr, T. A. Ostler, J. Barker, R. F. L. Evans, R. W. Chantrell, A. Tsukamoto, A. Itoh, A. Kirilyuk, T. Rasing, and A. V. Kimel, Nature (London) 472, 205 (2011).

[8] B. Vodungbo, B. Tudu, J. Perron, R. Delaunay, L. Müller, M. H. Berntsen, G. Grübel, G. Malinowski, C. Weier, J. Gautier, G. Lambert, P. Zeitoun, C. Gutt, E. Jal, A. H. Reid, P. W. Granitzka, N. Jaouen, G. L. Dakovski, S. Moeller, M. P. Minitti, A. Mitra, S. Carron, B. Pfau, C. von Korff Schmising, M. Schneider, S. Eisebitt, and J. Lüning, Sci. Rep. 6, 18970 (2016).

[9] S. Gerber, S.-L. Yang, D. Zhu, H. Soifer, J. A. Sobota, S. Rebec, J. J. Lee, T. Jia, B. Moritz, C. Jia, A. Gauthier, Y. Li,
D. Leuenberger, Y. Zhang, L. Chaix, W. Li, H. Jang, J.-S. Lee, M. Yi, G. L. Dakovski, S. Song, J. M. Glownia, S. Nelson, K. W. Kim, Y.-D. Chuang, Z. Hussain, R. G. Moore, T. P. Devereaux, W.-S. Lee, P. S. Kirchmann, and Z.-X. Shen, Science 357, 71 (2017).

[10] L. Chaix, G. Ghiringhelli, Y. Y. Peng, M. Hashimoto, B. Moritz, K. Kummer, N. B. Brookes, Y. He, S. Chen, S. Ishida, Y. Yoshida, H. Eisaki, M. Salluzzo, L. Braicovich, Z.-X. Shen, T. P. Devereaux, and W.-S. Lee, Nat. Phys. 13, 952 (2017).

[11] T. Wang, D. Zhu, B. Wu, C. Graves, S. Schaffert, T. Rander, L. Müller, B. Vodungbo, C. Baumier, D. P. Bernstein, B. Bräuer, V. Cros, S. de Jong, R. Delaunay, A. Fognini, R. Kukreja, S. Lee, V. López-Flores, J. Mohanty, B. Pfau, H. Popescu, M. Sacchi, A. B. Sardinha, F. Sirotti, P. Zeitoun, M. Messerschmidt, J. J. Turner, W. F. Schlotter, O. Hellwig, R. Mattana, N. Jaouen, F. Fortuna, Y. Acremann, C. Gutt, H. A. Dürr, E. Beaurepaire, C. Boeglin, S. Eisebitt, G. Grübel, J. Lüning, J. Stöhr, and A. O. Scherz, Phys. Rev. Lett. 108, 267403 (2012).

[12] M. Buzzi, M. Makita, L. Howald, A. Kleibert, B. Vodungbo, P. Maldonado, J. Raabe, N. Jaouen, H. Redlin, K. Tiedtke, P. M. Oppeneer, C. David, F. Nolting, and J. Lüning, Sci. Rep. 7, 7253 (2017).

[13] C. T. Chen, Y. U. Idzerda, H.-J. Lin, N. V. Smith, G. Meigs, E. Chaban, G. H. Ho, E. Pellegrin, and F. Sette, Phys. Rev. Lett. 75, 152 (1995).

[14] S. Valencia, A. Gaupp, W. Gudat, H.-C. Mertins, P. M. Oppeneer, D. Abramsohn, and C. M. Schneider, New J. Phys. 8, 254 (2006).

[15] K. Ramasesha, S. R. Leone, and D. M. Neumark, Annu. Rev. Phys. Chem. 67, 41 (2016).

[16] S. C. Nguyen, Q. Zhang, K. Manthiram, X. Ye, J. P. Lomont, C. B. Harris, H. Weller, and A. P. Alivisatos, ACS Nano 10, 2144 (2016).

[17] M. E. Vaida, B. M. Marsh, and S. R. Leone, Nano Lett. 18, 4107 (2018).

[18] E. Beaurepaire, J.-C. Merle, A. Daunois, and J.-Y. Bigot, Phys. Rev. Lett. 76, 4250 (1996).

[19] C. D. Stanciu, F. Hansteen, A. V. Kimel, A. Kirilyuk, A. Tsukamoto, A. Itoh, and T. Rasing, Phys. Rev. Lett. 99, 047601 (2007).

[20] F. Capotondi, E. Pedersoli, N. Mahne, R. H. Menk, G. Passos, L. Raimondi, C. Svetina, G. Sandrin, M. Zangrando, M. Kiskinova, S. Bajt, M. Barthelmess, H. Fleckenstein, H. N. Chapman, J. Schulz, J. Bach, R. Frömter, S. Schleitzer, L. Müller, C. Gutt, and G. Grübel, Rev. Sci. Instrum. 84, 051301 (2013).

[21] E. Allaria, R. Appio, L. Badano, W. A. Barletta, S. Bassanese, S. G. Biedron, A. Borga, E. Busetto, D. Castronovo, P. Cinquegrana, S. Cleva, D. Cocco, M. Cornacchia, P. Craievich, I. Cudin, G. D'Auria, M. Dal Forno, M. B. Danailov, R. De Monte, G. De Ninno, P. Delgiusto, A. Demidovich, S. 
Di Mitri, B. Diviacco, A. Fabris, R. Fabris, W. Fawley, M. Ferianis, E. Ferrari, S. Ferry, L. Froehlich, P. Furlan, G. Gaio, F. Gelmetti, L. Giannessi, M. Giannini, R. Gobessi, R. Ivanov, E. Karantzoulis, M. Lonza, A. Lutman, B. Mahieu, M. Milloch, S. V. Milton, M. Musardo, I. Nikolov, S. Noe, F. Parmigiani, G. Penco, M. Petronio, L. Pivetta, M. Predonzani, F. Rossi, L. Rumiz, A. Salom, C. Scafuri, C. Serpico, P. Sigalotti, S. Spampinati, C. Spezzani, M. Svandrlik, C. Svetina, S. Tazzari, M. Trovo, R. Umer, A. Vascotto, M. Veronese, R. Visintini, M. Zaccaria, D. Zangrando, and M. Zangrando, Nat. Photon. 6, 699 (2012).

[22] Theoretically the Co absorption edge is at $20.8 \mathrm{~nm}$ and we start the experiment at this wavelength but by measuring the magnetic contrast we observed that the best magnetic contrast was achieved for a wavelength of $20.5 \mathrm{~nm}$.

[23] P. Finetti, H. Höppner, E. Allaria, C. Callegari, F. Capotondi, P. Cinquegrana, M. Coreno, R. Cucini, M. B. Danailov, A. Demidovich, G. De Ninno, M. Di Fraia, R. Feifel, E. Ferrari, L. Fröhlich, D. Gauthier, T. Golz, C. Grazioli, Y. Kai, G. Kurdi, N. Mahne, M. Manfredda, N. Medvedev, I. P. Nikolov, E. Pedersoli, G. Penco, O. Plekan, M. J. Prandolini, K. C. Prince, L. Raimondi, P. Rebernik, R. Riedel, E. Roussel, P. Sigalotti, R. Squibb, N. Stojanovic, S. Stranges, C. Svetina, T. Tanikawa, U. Teubner, V. Tkachenko, S. Toleikis, M. Zangrando, B. Ziaja, F. Tavella, and L. Giannessi, Phys. Rev. X 7, 021043 (2017).

[24] The recorded transmission curves, as well as the XMCD spectrum, are given in arbitrary units because (i) we do not know precisely the conversion of CCD counts to photon number and (ii) we cannot measure the attenuation by the sample since without sample the CCD camera would be saturated.

[25] J. Stöhr and H. C. Siegmann, Magnetism: From Fundamentals to Nanoscale Dynamics, Springer Series in Solid-State Sciences (Springer, Berlin, 2006).

[26] A signal as small as $0.7 \%$ can be measured for 500 shots which corresponds to an acquisition time of $50 \mathrm{~s}$. Taking into account the CCD reading time and the set of four pictures and two magnetization, the total experimental time to get the XMCD signal is $20 \mathrm{~min}$. This compares to a total experimental time of 12 min for single-shot data.

[27] S. Alebrand, U. Bierbrauer, M. Hehn, M. Gottwald, O. Schmitt, D. Steil, E. E. Fullerton, S. Mangin, M. Cinchetti, and M. Aeschlimann, Phys. Rev. B 89, 144404 (2014).

[28] R. Medapalli, I. Razdolski, M. Savoini, A. R. Khorsand, A. Kirilyuk, A. V. Kimel, T. Rasing, A. M. Kalashnikova, A. Tsukamoto, and A. Itoh, Phys. Rev. B 86, 054442 (2012).

[29] N. Beaulieu, Theses, Université Paris Sud - Paris XI, 2013.

[30] G. Malinowski, F. Dalla Longa, J. H. H. Rietjens, P. V. Paluskar, R. Huijink, H. J. M. Swagten, and B. Koopmans, Nat. Phys. 4, 855 (2008).

[31] We were able to measure a total time window of $1.2 \mathrm{ps}$, but because of border effect, IR leak, and shadows of higher diffraction order of the zone plate, the data are meaningful only from -0.2 to $0.6 \mathrm{ps}$.

[32] T. Ferté, N. Bergeard, L. Le Guyader, M. Hehn, G. Malinowski, E. Terrier, E. Otero, K. Holldack, N. Pontius, and C. Boeglin, Phys. Rev. B 96, 134303 (2017).

[33] C. Stamm, T. Kachel, N. Pontius, R. Mitzner, T. Quast, K. Holldack, S. Khan, C. Lupulescu, E. F. Aziz, M. Wietstruk, H. A. Dürr, and W. Eberhardt, Nat. Mater. 6, 740 (2007).
[34] C. Boeglin, E. Beaurepaire, V. Halté, V. López-Flores, C. Stamm, N. Pontius, H. A. Dürr, and J.-Y. Bigot, Nature (London) 465, 458 (2010).

[35] B. Koopmans, G. Malinowski, F. Dalla Longa, D. Steiauf, M. Fähnle, T. Roth, M. Cinchetti, and M. Aeschlimann, Nat. Mater. 9, 259 (2010).

[36] M. Battiato, K. Carva, and P. M. Oppeneer, Phys. Rev. Lett. 105, 027203 (2010).

[37] B. Pfau, S. Schaffert, L. Müller, C. Gutt, A. Al-Shemmary, F. Büttner, R. Delaunay, S. Düsterer, S. Flewett, R. Frömter, J. Geilhufe, E. Guehrs, C. M. Günther, R. Hawaldar, M. Hille, N. Jaouen, A. Kobs, K. Li, J. Mohanty, H. Redlin, W. F. Schlotter, D. Stickler, R. Treusch, B. Vodungbo, M. Kläui, H. P. Oepen, J. Lüning, G. Grübel, and S. Eisebitt, Nat. Commun. 3, 1100 (2012).

[38] B. Vodungbo, J. Gautier, G. Lambert, A. B. Sardinha, M. Lozano, S. Sebban, M. Ducousso, W. Boutu, K. Li, B. Tudu, M. Tortarolo, R. Hawaldar, R. Delaunay, V. López-Flores, J. Arabski, C. Boeglin, H. Merdji, P. Zeitoun, and J. Lüning, Nat. Commun. 3, 999 (2012).

[39] C. La-O-Vorakiat, E. Turgut, C. A. Teale, H. C. Kapteyn, M. M. Murnane, S. Mathias, M. Aeschlimann, C. M. Schneider, J. M. Shaw, H. T. Nembach, and T. J. Silva, Phys. Rev. X 2, 011005 (2012).

[40] D. Rudolf, C. La-O-Vorakiat, M. Battiato, R. Adam, J. M. Shaw, E. Turgut, P. Maldonado, S. Mathias, P. Grychtol, H. T. Nembach, T. J. Silva, M. Aeschlimann, H. C. Kapteyn, M. M. Murnane, C. M. Schneider, and P. M. Oppeneer, Nat. Commun. 3, 1037 (2012).

[41] N. Bergeard, V. López-Flores, V. Halté, M. Hehn, C. Stamm, N. Pontius, E. Beaurepaire, and C. Boeglin, Nat. Commun. 5, 3466 (2014).

[42] J. Wieczorek, A. Eschenlohr, B. Weidtmann, M. Rösner, N. Bergeard, A. Tarasevitch, T. O. Wehling, and U. Bovensiepen, Phys. Rev. B 92, 174410 (2015).

[43] E. Jal, V. López-Flores, N. Pontius, T. Ferté, N. Bergeard, C. Boeglin, B. Vodungbo, J. Lüning, and N. Jaouen, Phys. Rev. B 95, 184422 (2017).

[44] W. Zhang, W. He, X.-Q. Zhang, Z.-H. Cheng, J. Teng, and M. Fähnle, Phys. Rev. B 96, 220415(R) (2017).

[45] P. Tengdin, W. You, C. Chen, X. Shi, D. Zusin, Y. Zhang, C. Gentry, A. Blonsky, M. Keller, P. M. Oppeneer, H. C. Kapteyn, Z. Tao, and M. M. Murnane, Sci. Adv. 4, eaap9744 (2018).

[46] W. You, P. Tengdin, C. Chen, X. Shi, D. Zusin, Y. Zhang, C. Gentry, A. Blonsky, M. Keller, P. M. Oppeneer, H. Kapteyn, Z. Tao, and M. Murnane, Phys. Rev. Lett. 121, 077204 (2018)

[47] P. Hansen, S. Klahn, C. Clausen, G. Much, and K. Witter, J. Appl. Phys. 69, 3194 (1991).

[48] J. Hohlfeld, E. Matthias, R. Knorren, and K. H. Bennemann, Phys. Rev. Lett. 78, 4861 (1997).

[49] T. Roth, A. J. Schellekens, S. Alebrand, O. Schmitt, D. Steil, B. Koopmans, M. Cinchetti, and M. Aeschlimann, Phys. Rev. X 2, 021006 (2012).

[50] U. Atxitia, O. Chubykalo-Fesenko, J. Walowski, A. Mann, and M. Münzenberg, Phys. Rev. B 81, 174401 (2010).

[51] O. J. Suarez, P. Nieves, D. Laroze, D. Altbir, and O. ChubykaloFesenko, Phys. Rev. B 92, 144425 (2015). 
[52] U. Atxitia, J. Barker, R. W. Chantrell, and O. ChubykaloFesenko, Phys. Rev. B 89, 224421 (2014).

[53] N. Moisan, G. Malinowski, J. Mauchain, M. Hehn, B. Vodungbo, J. Lüning, S. Mangin, E. E. Fullerton, and A. Thiaville, Sci. Rep. 4, 4658 (2014).
[54] J. Mendil, P. Nieves, O. Chubykalo-Fesenko, J. Walowski, T. Santos, S. Pisana, and M. Münzenberg, Sci. Rep. 4, 3980 (2014).

[55] K. C. Kuiper, T. Roth, A. J. Schellekens, O. Schmitt, B. Koopmans, M. Cinchetti, and M. Aeschlimann, Appl. Phys. Lett. 105, 202402 (2014). 